\title{
PHOSPHOMOLYBDENUM BLUE DETECTION - A REVIEW OF CHARACTERISTICS, ACHIEVEMENTS, CHALLENGES AND FUTURE PROSPECTS
}

\author{
G. N. Doku*, W. K. Agbozo, S. J. Haswell And T. Mccreedy \\ (G. N. D. \& W. K. A.: School of Applied Sciences, Central University, Miotso, Ghana; S. J. \\ H. \& T. M.: Department of Chemistry, University of Hull, HU6 7RX, UK). \\ *Corresponding author's email: gndoku@hotmail.com
}

\begin{abstract}
Phosphate detection in the environment (especially, water bodies) is very essential in view of its consequent pollution effects on eutrophication. Continuous monitoring of phosphate concentration (and phosphorus compounds in general) in water samples has been based on phosphomolybdenum blue formation coupled with spectrophotometric detection. Continuous flow injection analyses (FIA) are well known to present numerous advantages over batch methods. Furthermore, the development of on-chip micro-channel analytical ( $\mu$ FIA) systems begun and have gained much attention within the last two decades in view of the striking advantages over conventional FIA techniques. This paper reviews published information on detection of phosphomolybdenum blue in conventional systems as well as on micro-chip. It reports on the challenges encountered (interference from other complex anions), the achievements made so far (intereference removal by electrokinetic separation) and what the future holds (simultaneous determination).
\end{abstract}

Keywords: Phosphate, phosphomolybdenum blue, on-microchip, analysis, review.

\section{Introduction}

While being an essential nutrient for phytoplankton in aquatic systems, phosphorus in excessive concentration can trigger abnormal growth of algae in lakes and coastal sea and result in eutrophication or even red tide. As phosphorus at concentrations over $20 \mu \mathrm{g} / \mathrm{l}$ can accelerate eutrophication, the lowest determinable phosphorus concentration should be in the range of a few $\mu \mathrm{g} / \mathrm{L}$ (Warwick et al., 2013; Yeh et al., 2017). There has always been the demand for detectors/sensors that would accurately and reliably determine phosphate concentrations and ideally allow real-time monitoring. Molybdenum blue formation coupled with spectrophotometric detection has been the standard method employed for ages, typically for the continuous monitoring of phosphate concentration (and phosphorus compounds in general) in water samples (Ruzicka et al., 1988; Verporte et al., 1994; Clark et al., 1992; Robards et al., 1994). The method is based on an absorption maxima at 880, 825, 774 and 660 $\mathrm{nm}$ wavelengths at which absorbance is strong for the molybdenum blue complex which has a molar extinction coefficient $(\varepsilon) \geq 14,300 \mathrm{~L} /$ (mole.cm) (Ruzicka et al., 1988; Verporte et al., 1994). The reagents employed are ammonium molybdate $\left.\left[\left(\mathrm{NH}_{4}\right)_{6} \mathrm{Mo}_{7} \mathrm{O}_{24} \cdot 4 \mathrm{H}_{2} \mathrm{O}\right)\right]$, L-ascorbic acid and the phosphate analyte solution(s). The reactions involved are as follows: 


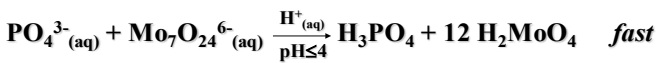

$$
\begin{aligned}
& \mathrm{H}_{3} \mathrm{PO}_{4}+12 \mathrm{H}_{2} \mathrm{MoO}_{4} \longrightarrow \underset{(12 \mathrm{MPA})}{\longrightarrow} \mathrm{H}_{3} \mathrm{PO}_{4}\left(\mathrm{MoO}_{3}\right)_{12},+12 \mathrm{H}_{2} \mathrm{O} \text { fast } \\
& \text { Mo(VI) } \underset{\text { (reduction) }}{\stackrel{\text { Ascorbicacid }}{\longrightarrow}} \underset{\text { (PMB) }}{\operatorname{Mo}(\text { V) }}
\end{aligned}
$$

A typical challenge with the molybdenum blue technique is that other trace complex anions are also associated with molybdenum blue chemistry, and would therefore interfere with the phosphate detection; these complex anions include arsenate (As(III) and $\mathrm{As}(\mathrm{V})$ ), silicate, vanadate, tungstate and others such as germinate, which are also potential toxicants to the environment (Doku et al., 1999; Basset et al., 1978). The interfering molybdo-complexes are $\mathrm{H}_{3} \mathrm{PO}_{4}\left(\mathrm{MoO}_{3}\right)_{12}, \mathrm{H}_{4} \mathrm{SiO}_{4}\left(\mathrm{MoO}_{3}\right)_{12}$, $\mathrm{H}_{3} \mathrm{As} \mathrm{O}_{4}\left(\mathrm{MoO}_{3}\right)_{12}, \mathrm{H}_{3} \mathrm{~V} \mathrm{O}_{4}\left(\mathrm{M} \mathrm{oO} \mathrm{O}_{3}\right)_{12}$, $\mathrm{H}_{2} \mathrm{WO}_{4}\left(\mathrm{MoO}_{3}\right)_{12}$, and $\mathrm{H}_{4} \mathrm{GeO}_{4}\left(\mathrm{MoO}_{3}\right)_{12}$ (Ruzicka et al., 1988; Verporte et al., 1994; Doku et al., 1999; Clark et al., 1992; Robards et al., 1994; Basset et al., 1978). The prior isolation of interfering species, to make way for the selective detection of one particular species of interest, has employed conventional manual batch procedures that make exorbitant use of expensive and corrosive reagents and are often labour intensive and time consuming (Basset et al., 1978; Mattusch, 1996; Demedina et al., 1996; Bello et al., 1996; Motomizu et al., 1995; Galceran et al., 1994). Procedures such as liquid-liquid extraction of phosphate from sulphate and silicate interferences (Mattusch et al., 1996), ion chromatographic separation of phosphate from silicate, sulphate and sulphites (Demedina et al., 1996; Bello et al., 1996), filtration-dissolution (Motomizu et al., 1995) and preconcentration methods for ultra-trace phosphate analysis (Galceran et al., 1994) have already been reported. The molybdenum blue method is also susceptible to refractive index errors and sample turbidity (Warwick et al., 2013; Udnan et al., 2005). This paper assesses other phosphate detection methods reported in literature and, more importantly, reviews phosphomolybdenum blue techniques that have been achieved, how the interference challenges had been dealt with and what is probable for the future.

\section{Experimental}

Other phosphate detection methods reported in literature

Methods other than phosphomolybdenum blue-spectrophotometry that have been researched and reported so far for inorganic phosphate detection are optical and electrochemical.

\section{Optical detection methods}

The optical detection methods reported for inorganic phosphate detection have employed biomimetic phosphate sensor/receptor methodologies. Phosphate sensors/receptors are molecular moieties specially designed and synthesized to selectively recognize and bind to the phosphate anion so it can be detected and quantified. The receptors include synthetic receptors, molecularly imprinted polymers (MIPs) and others (Warwick et al., 2013). The use of phosphate sensors/receptors, coupled with optical detection has been researched quite extensively. The detection has normally been colorimetric (Tobey \& Anslyn, 2003; Han \& Kim, 2002) and luminescence/fluorescence (Yaqoob et al., 2004; Lin et al., 2006; Sun et al., 2008; Ganjali et al., 2011; Anzenbacher et al., 2000; Beer \& Cadman, 1999; Ojida et al., 2002, 2004; Hamachi, 2009; Sessler et al., 2003; Deetz \& Smith, 1998; Du et al., 2008; Fabbrizzi et al., 2002). These methods have yielded detection limits varying from milligrams to micrograms per litre but which 
are higher than the much lower detection limits required for environmental applications. The receptors are also too complex to design and synthesize and they have the problem of selectivity for phosphate (interference from other anions - sulphate, chloride, nitrate, acetate, etc.) and require integration with appropriate transducers. There is also the problem of functionality in aqueous media (in contrast to organic-based media, due to the high hydration energy of the phosphate anion) and there is always fouling from solid particles and naturally occurring organic matter in water samples. All these disadvantages have limited their development and suitability for long term field use. Yaqoob et al. (2004) successfully adapted the phosphomolybdenum blue method to incorporate luminol which, when oxidised by the phosphomolybdate complex, generated light via chemiluminesenc. The method could detect phosphates in freshwater samples with a detection limit of $0.03 \mu \mathrm{g} / 1$ and a linear response from 0.03 to $3.26 \mu \mathrm{g} / \mathrm{l}$, making it suitable for monitoring natural environments with little anthropogenic activity or pollution, but not for the wide range of phosphate concentrations found in wastewater $(100-5000 \mu \mathrm{g} / \mathrm{l})$. Competing ions were removed by passing samples through a chelating resin, significantly reducing interference. However, copper (II), cobalt, magnesium and iron (II) ions suppressed or exaggerated the signal produced, by acting as catalysts for the oxidation of luminol (Warwick et al., 2013; Yaqoob et al., 2004). The same disadvantage was observed in a PVC membrane containing an aluminium-morin fluorescent probe, as nickel and zinc ions caused interference by accelerating the quenching process (Lin et al., 2006). The latter presented a response time of 7.5 minutes, detection limit of $0.02 \mu \mathrm{g} / \mathrm{l}$ and linear response to concentrations of dihydro- gen phosphate from 6 to $15 \mu \mathrm{g} / \mathrm{l}$. Although the response was reversible, by applying more aluminium-morin, the probe was only viable for sixty measurements, limiting its suitability for continuous monitoring (Lin et al., 2006).

\section{Electrochemical sensor methodologies for phosphate detection}

The employment of various electrodes and electrochemical techniques (potentiometric, voltammetric and amperometric methods) in orthophosphate detection has been reviewed extensively (Warwick et al., 2013; Midgley, 1986; Engblom, 1998 and Villalba et al., 2009).

The potentiometric methods have involved the use of metal electrodes and phosphate ion selective membranes (Antonisse and Reinhoudt, 1999; Tsagkatakis et al., 2001; Glazier and Arnold, 1988; Liu et al., 1997; Carey and Riggan, 1994; Le Goff et al., 2004; Nishizawa et al., 2003; Modi et al., 2011; Liu et al., 2007; Ganjali et al., 2006; Kumar et al., 2008). Solid state ion selective detection offers numerous advantages, including increased opportunity for miniaturisation, greater durability and the elimination of the need for a reference storage solution, which incurs cost and requires regular maintenance, as the reagents get consumed, evaporate or leak (Kumar et al., 2010; Engblom, 1998; Ejhieha \& Masoudipoura, 2010; Tafesse \& Enemchukwu, 2011). Solid state ion selective methods yielded quick response times (seconds), linear response range 0.016 $-3100 \mathrm{mg} / \mathrm{L}$ and detection limits as low as $0.024 \mathrm{mg} / \mathrm{L}$ (Ejhieha \& Masoudipoura, 2010; Kumar et al., 2010; Xiao et al., 1995; Meruva \& Meyerhoff, 1996; Zou et al., 2007; Lee et al., 2009; Khaled et al., 2008; Kugimiya \& Kohara, 2009). While yielding good detection limits and linear ranges near that required for environmental monitoring, many potentiomet 
ric methods suffer from the general difficulty of developing phosphate selective electrodes, suitability for anions, the high hydrophilic nature of the phosphate anion, limited durability (linear ranges deteriorate after few weeks) and/or poor selectivity from interfering ions (Warwick et al., 2013). The electrodes may also require pre-treatment in phosphate solution for 24 hours, reducing the frequency of readings that could be taken in the field (Ganjali et al., 2006).

The use of Amperometric sensors, that measure the current produced by the target analyte at an electrode with a constant voltage, has also been investigated widely (Beer et al., 1996, 1997, 1999) showing some selectivity for dihydrogen phosphate and orthophosphate. Some involved measuring the reduction of the ammonium phosphomolybdate complex formed in the standard phosphomolybdate reaction (Quintana et al., 2004; Udnan et al., 2005) or anodic oxidation of molybdenum (Jonca et al., 2011), while others involved the use of electrodes embedded with ammonium molybdate (Berchmans et al., 2011). Others involved the use of phosphate sensitive enzyme electrodes, designed for potential environmental applications, and are able to detect orthophosphates down to $3 \mu \mathrm{g} / \mathrm{L}$ (Midgley, 1986; Engblom, 1998; Villalba et al., 2009; Rahman et al., 2006). The amperometric methods however suffer from the need for reagents that require safe disposal, lack of specificity for phosphates (without interference from other ions - arsenate, chloride or nitrate, silicate, sulphate, etc.) and the fact that none of the amperometric methods offer detection covering the range of phosphate typically found in wastewater $(0.1-5 \mathrm{mg} / \mathrm{L})$. Furthermore, besides uncertain stability and doubts regarding durability, the production of enzyme-based biosensor electrodes is more expensive than other sensor-based technologies (Zou et al., 2007).

Some conductometric analysis of phosphate has been reported (Warwick et al., 2013; Zhang et al., 2008), producing two linear ranges ( 0.03 $-0.62 \mathrm{mg} / \mathrm{L}$ and $0.62-12.4 \mathrm{mg} / \mathrm{L}$ ), detection limit of $0.03 \mathrm{mg} / \mathrm{L}$ and the sensor showed no interference from other anions. However, sensitivity to both $\mathrm{pH}$ and temperature (optimum conditions pH 6 and $20-50^{\circ} \mathrm{C}$ ), resulting in a $50 \%$ reduction in response after three weeks, makes the system unsuitable for field analysis (Warwick et al., 2013).

The use of voltammetric devices to measure orthophosphate activity is largely inhibited by the oxygen ions around the central phosphorus atom. Voltammetric analysis of orthophosphates has therefore tended to rely on indirect methods, for example, measuring the reduction of the ammonium phosphomolybdate complex formed in the standard phosphomolybdate reaction (Fogg \& Bsebsu, 1981), using receptors coated on electrodes to cause measurable indirect reactions (Aoki et al., 2003; Beer et al., 1995; Liu and Lin, 2005; Cheng et al., 2010). While detection limits as low as $9 \mu \mathrm{g} / \mathrm{L}$ to $0.03 \mathrm{mg} / \mathrm{L}$ and linear response between 1.2 and $31 \mathrm{mg} / \mathrm{L}$ have been achieved using voltammetry with flow injection analysis (FIA), they suffer from regular cleaning of electrodes, selectivity for inorganic phosphate anion (interference from $\mathrm{SO}_{4}^{2-}, \mathrm{Br}, \mathrm{Cl}^{-}$, etc.) and suitability for field environmental applications due to the indirect nature and requirement for more volumes of other reagents than in the standard colourimetric method (Warwick et al., 2013).

The need for increased monitoring of phosphate levels in environmental water requires a cost-effective sensor that is both specific for phosphate in a complex medium, and robust enough to withstand field usage, in or- 
der to be a suitable replacement for standard measurement methods. While the luminescent/fluorescent methods reviewed have low detection limits and could potentially measure phosphate concentrations at the limit $(1 \mathrm{mg} / \mathrm{l})$ required by Urban Waste Water Treatment Directives, none of them are both specific for phosphate and have been successfully tested in the field with environmental samples. Furthermore, the methods reviewed produce their signal in solution, again requiring the consumption and disposal of reagents. Generally, from the electrochemical methods reviewed, the ISEs suffer from limited lifetimes; require pre-treatment in solution, or both, limiting suitability for field measurements and continuous monitoring. Similarly, solid state potentiometric sensors suffer from poor selectivity or durability. The voltammetric and amperometric sensors suffer from various obstacles, mainly a lack of selectivity, limiting their suitability and while enzyme based amperometric sensors may perform well in biomedical applications, their suitability and robustness for environmental field use is doubtful (Warwick et al., 2013). These issues highlight why no sensor-based technology has, so far, been validated to replace the standard colourimetric technique for determining the phosphate content of environmental water.

Conventional phosphoolybdenum blue detection procedures

In any batch procedure, ammonium molybdate is added to orthophosphate samples with ascorbic acid and antimony (III), which produces the blue coloured phosphomolybdate complex. The concentration of phosphate, contained in a cuvette, is then determined optically with a spectrophotometer (Murphy \& Riley, 1962). The conventional continuous flow injection analysis (FIA) manifold for phosp- hate molybdebum blue (PMB) detection is that shown in Figure 1 (Ruzicka et al., 1988).

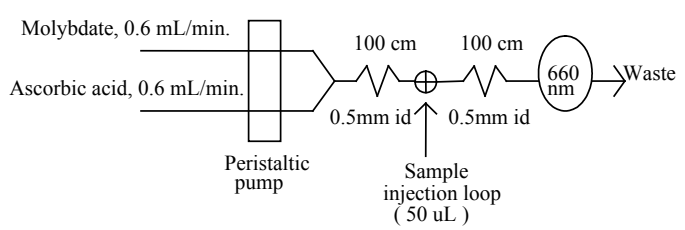

Fig. 1: Conventional FIA manifold for the spectrophotometric determination of phosphate.

Commercially available phosphomolybdenum blue methods either involve measurement on site or require samples sent away for analysis, with both approaches being labour intensive and require continued use of consumables (reagents and cuvettes), which incur direct costs and require safe disposal. Today's research is geared towards having a detector/sensor device which can measure soluble phosphate in water and which would incur significant cost savings, particularly if it was designed to provide in situ, continuous (on-line) and real-time monitoring.

On-chip micro-channel phosphomolybdenum blue flow injection analysis ( $\mu$ FIA)

The development of on-chip micro-channel analytical $(\mu$ FIA) systems begun and have gained much attention within the last two decades. The most striking advantages of such microsystems are the high portability, reduced reagent consumption, minimization of waste production, remote (on-site) applications and heat dissipation owing to the high surface area to volume ratios ((Verporte et al., 1994; Greenway et al., 1999; Natalie et al., 2000; Reyes et al., 2002; Auroux et al., 2002; Daykin et al., 1995; Haswell, 1997; Irfan et al., 2011; Wegner et al., 2011; Costantini et al., 2010; Costantini et al., 2010; Frost et al., 2010; Wiles et al., 2012). 
Verporte et al. (1994) first described a three-dimensional $\mu$ FIA system $\left(22 \times 22 \mathrm{~mm}^{2}\right.$ planar micromachined silicon elements), based on a conventional FIA manifold (Figure 1) for phosphate detection. The system employed externally connected piezoelectrically driven silicon micropumps for reagent mobilization, operating at flow rates of $0.03-0.2 \mathrm{~mL} / \mathrm{min}$ which correspond to about one-tenth of those used in a conventional FIA system, leading to a corresponding 270 -fold reduction in reagent consumption. Sample volumes used with the microflow system were around $25 \mu \mathrm{L}$. To increase the sensitivity of the technique, since the analysis is carried out under non-equilibrium conditions, a stopped-flow mode was adopted, producing an analysis time of $4 \mathrm{~min}$ per sample. The sensitivity achieved with the $\mu$ FIA system was comparable to that expected for a conventional system $\left(5 \mu \mathrm{gmL}^{-1}\right.$ phosphates as orthophosphate).

In an attempt to exploit electroosmotic flow (EOF) rather than the hydrodynamic flow as described by Verporte et al., (1994), Daykin et al., (1995) described the development of a $\mu$ FIA system, also for the determination of orthophosphate. ChDannels $325 \mu \mathrm{m}$ wide and $30 \mu \mathrm{m}$ deep were etched into a glass substrate in which molybdate ( $\mathrm{pH} 3.2$ ) was mobilized by EOF; however, due to $\mathrm{pH}$ constraints (pH 1.6), it was necessary to pump the ascorbic acid by ion migration rather than EOF. The reagents were combined in a contra-flow manner and a Z-mode injection (Haswell, 1997) was used to introduce the orthophosphate. On completion of the molybdenum blue reaction, the product was detected in situ using a fibre-optic configuration. The authors reported an RSD of $<5 \%$ at $10 \mathrm{ppb}$, decreasing to $<0.8 \%$ at $100 \mathrm{ppb}$ for orthophosphate, where $n=5$. The linear working range was found to be $10-100 \mathrm{ppb}$, and the system produced a limit of detection of 0.7 ppb.

In a further study, Doku et al. (1999) reported on the development of a micro-FIA ( $\mu$ FIA) system based on electroosmotic flow the determination of phosphate as orthophosphate. Photolithographic and wet etching techniques were used to fabricate a $\mu$ FIA manifold (Figure 2) in a borosilicate glass substrate for the spectrophotometric determination of orthophosphate based on the molybdenum blue reaction, employing electroosmotic flow for both the mobilization of reagents and sample injection. The manifold channel dimensions were $200 \mu \mathrm{m}$ wide and $50 \mu \mathrm{m}$ deep. An evaluation of the electroosmotic flow characteristics (i.e. voltage, solution concentration/pH ranges and current-voltage relationships) for ascorbic acid, ammonium molybdate and orthophosphate standards, with and without borate buffer present, were carried out. In addition, the effect that reaction precursors may have on the in situ spectrophotometric detection system is also discussed. Using the optimized operating conditions of $1.75 \%$ ascorbic acid and $0.6 \%$ ammonium molybdate in $10 \mathrm{mM}$ borate buffer, the calibration model for phosphate standards was found to be linear $(y=0.0036 x+0.0155$; correlation coefficient, $\mathrm{r}^{2}=0.9952$ ) over the working range 1-10 $\mu \mathrm{gmL}^{-1}$. A sampling rate of ca. 60 samples/h was achieved, with a detection limit of $0.1 \mu \mathrm{gmL}^{-1}$ for orthophosphate, with RSDs $<5 \%$. The reagent consumption was very low with the total reactant volume in the system estimated to be $0.6 \mu \mathrm{L}$ and a sample volume of $0.1 \mu \mathrm{L}$ being required. 


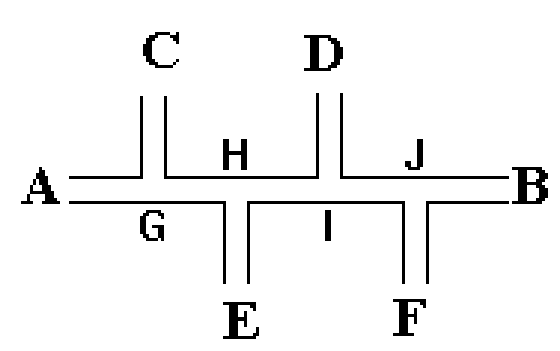

Fig. 2: $\mu$ FIA manifold for determining flow characteristics of reagents (channels: $200 \mu \mathrm{m}$ wide, $50 \mu \mathrm{m}$ deep). $\mathrm{A}=$ reagent reservoir, $\mathrm{B}=$ waste reservoir and $\mathrm{C}-\mathrm{F}=$ side channel reservoirs. $\mathrm{AB}=18 \mathrm{~mm}, \mathrm{AG}=\mathrm{GH}$ $=\mathrm{HI}=\mathrm{IJ}=\mathrm{JB}=3.5 \mathrm{~mm}, \mathrm{CG}=\mathrm{EH}=\mathrm{DI}=\mathrm{FJ}=8 \mathrm{~mm}$.

Clinton-Bailey et al. (2017) have described a robust, high resolution in situ sensor suited to in situ work in natural waters, where high sensitivity and precision are required. It features an improved "phosphate blue" assay which includes polyvinylpyrrolidone in place of traditional surfactants - improving sensitivity and reducing temperature effects. The sensor is housed in a circular hardware, which is based around a circular tinted PMMA microfluidic chip. It operates using a stop-flow regime, whereby sample and reagents are introduced into the absorbance flow cell and then left to react (and color to develop) over a set period. The reaction time was set at $5 \mathrm{~min}$, with the average voltage over the final $5 \mathrm{~s}$ of the development time used to determine absorbance. The system featured greater power economy and analytical performance relative to commercially available alternatives, with a mean power consumption of $1.8 \mathrm{~W}$, a detection limit of $40 \mathrm{nM}$, a dynamic range of $0.14-10 \mu \mathrm{M}$, and an infield accuracy of $4 \pm 4.5 \%$. During field testing, the sensor was continuously deployed for 9 weeks in a chalk stream, revealing complex relations between flow rates and phosphate concentration that suggest changing dominance in phosphate sources. A distinct diel phosphorus signal was observed under low flow conditions, highlighting the ability of the sensor to decouple geochemical and biotic effects on phosphate dynamics in fluvial environments. Antimonyl tartrate was used to catalyze the reaction and to inhibit the formation of silicomolybdic acid, which is a common interference of this assay. Removal of any of the other interference ions, if present, would however be required.

\section{Coupling external UV-Vis spectrophotometers} to microchip devices

The Lambert-Beer law states that absorption is proportional to the light pathlength and the concentration of the absorbing species. Microchips have small pathlengths, which could greatly reduce sensitivity and limits of detection (LOD). To increase the sensitivity by decreasing the background noise and lengthening the optical path, Gáspár et al employed a modified external micro-spectrophotometer, combined with a projection microscope, that precisely selects very small parts of a microfluidic channel pattern of disposable PDMS chips and measure light absorbance on a magnified area of the selected part of the channel. Besides the external direct detection above the microfluidic channel, the optical pathlength was lengthened by detecting in the region of a perpendicular exit port; increasing the cross-sectional area of the zone of irradiation improved the signal-to-noise ratio and the limits of detection (LOD) to as low as $0.78 \mu \mathrm{M}$ for phosphomolybdenum blue. The use of such a system in phosphomolybdenum detection would also require removal of interference from other related ions, if present.

\section{LED light sources in phophomolybdenum blue detection on-chip}

The potential of LEDs as effective light sources in chemical sensing devices have been reviewed by Yeh et al., (2017). LEDs give high light intensity but consume less power. LED 
light sources are stable, robust and inexpensive. LED-based sensors do not require optical couplers or monochromators since LEDs emit relatively narrow band of wavelength. They are commercially available in the spectral range of $210-1550 \mathrm{~nm}$. Using fibre optics, these LEDs can easily be coupled to photodiodes in microfluidic systems and they are long lasting even under unfavorable conditions like high humidity and mechanical vibration. LED has successfully been applied for fluorescence excitation in microfluidic devices for sensing gases, spotting heavy metal ions, analyzing drugs and toxins, for detecting environment nutrients (ammonia, Nitrite and total nitrate and phosphorus), and for monitoring biochemicals and biohazard. LED technologies are suitable for miniaturized, on-chip analytical systems as they can be integrated with microchip due to their small size. Red and blue LEDs, typically, have shown their versatilities in the fields of optical sensors and chemical sensing devices. Ma et al. (1999) built a portable micro flow injection system with a red LED $(\sim 660 \mathrm{~nm})$ for molybdenum blue detection. The system, with phosphorus limit of detection (LOD) at $5 \mu \mathrm{g} / 1$, was used for onsite phosphate detection in river water samples via the detection of molybdenum blue, a reduction product of molybdenum yellow. Both molybdenum blue and molybdenum yellow are formed via the reaction of orthophosphate, the most bioavailable phosphorus form, with molybdate in acidic medium. Lyddy-Meaney et al. (2002) developed a compact flow injection analysis system with a gas-pressure propelling system and a red LED $(\sim 650 \mathrm{~nm})$ for marine water phosphate surface mapping. This simple device applied molybdenum blue method to rapidly determine trace and ultra-trace amounts of phosphorus as waterborne phosphate demonstrated a LOD of $0.1 \mu \mathrm{g} / \mathrm{l}$. It is important to note, however, that the use of LED in phosphomolybdenum detec- tion would still require the removal of other related interfering ions, if present.

Effective and robust execution of molybdenum chemistry on-chip would require further studies into the interference capabilities of the various complex-anion species and to establish some surest ways of removing any interferences to make way for the determination of the species, coupled with intelligent/appropriate manifold designs. Detection techniques which do not involve molybdenum blue formation could also be considered. It would be more interesting to have procedures that could even execute simultaneous determination of the interfering species.

Assessing molybdenum interference capabilities among the complex-anion species

A recent work (Doku et al., 2016) presented an assessment of the relative rates of formation and relative intensities of the molybdenum blue complexes and an evaluation of the UV-visible absorbance profiles to ascertain the wavelengths of absorption and the extinction coefficients of the respective unreacted species as well as their corresponding blue molybdo complexes. These would reveal the potential interference capabilities of the species (arsenate, orthophosphate, silicate, vanadate and tungstate) in spectrophotometric determination based on molybdenum blue. Figure 3 shows the scanned UV-visible absorbance profiles obtained for the $1.75 \%$ ascorbic acid$0.6 \%$ ammonium molybdate reagent mixture, and the individual blue reaction products derived from the different interfering species. 


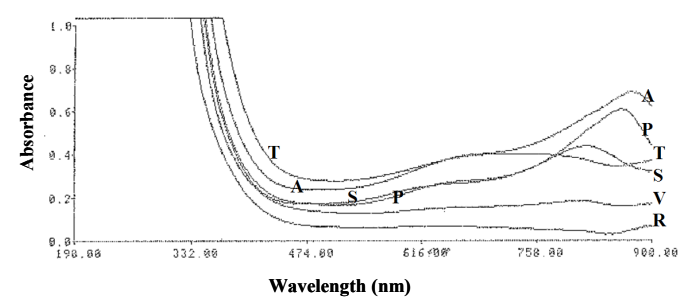

Fig. 3: Scanned UV-visible absorbance profiles for the molybdenum blue reaction products obtained from the different interfering species: $\mathrm{A}=$ arsenate, $\mathrm{P}=$ orthophosphate, $\mathrm{T}=$ tungstate, $\mathrm{S}=$ silicate, $\mathrm{V}=$ orthovanadate, and $\mathrm{R}=$ yellow-green $1.75 \%$ ascorbic acid- $0.6 \%$ ammonium molybdate reagent mixture.

Both the yellow-green background reagent mixture and the molybdenum blue reaction products from the different species showed similar absorbance profiles, absorbing in the whole UV-visible range from $190-900 \mathrm{~nm}$, with maximum absorbance at wavelengths less than $400 \mathrm{~nm}$ and reasonably strong absorbance between $600-900 \mathrm{~nm}$ (the visible wavelength range within which the species can be detected), but with different $\lambda_{\text {maxes }}$. The visible absorption extinction coefficients $(\xi)$ estimated for the molybdenum blue complexes of the species at respective ${ }_{\text {maxes }}$ obtained from Figure 3 were as follows: arsenate, $4.2635 \times 10^{3}$ $\mathrm{L} /($ mol.cm $)\left(\lambda_{\max } 880 \mathrm{~nm}\right)$; orthophosphate, $3.7470 \times 10^{3} \mathrm{~L} /(\mathrm{mol} . \mathrm{cm})\left(\lambda_{\max } 860 \mathrm{~nm}\right)$; silicate, $2.7132 \times 10^{3} \mathrm{~L} /(\mathrm{mol} . \mathrm{cm})\left(\lambda_{\max } 820 \mathrm{~nm}\right)$; tungstate, $2.4546 \times 10^{3} \mathrm{~L} /(\mathrm{mol} . \mathrm{cm})\left(\lambda_{\max } 687\right.$
- $774 \mathrm{~nm})$; and orthovanadate, $1.0983 \times 10^{3}$ $\mathrm{L} /(\mathrm{mol} . \mathrm{cm})\left(\lambda_{\max } 785-825 \mathrm{~nm}\right)$, indicating a decreasing absorbance ratio/trend of arsenate (3.88) : orthophosphate (3.42) : silicate (2.47) : tungstate (2.24) : orthovanadate (1.00). The above scanned visible absorbance profiles and extinction coefficient ratios were obtained using equal concentrations of the species so as to remove any biases, which may not directly correspond to the concentration levels of the species in 'real' environmental samples. The degree of interference in real sample analysis will also depend on the actual relative concentration and detection levels of the species in the environmental sample. Table 1 gives the concentration range (in ppb), as well as the major chemical form of occurrence, of the species in natural fresh water and sea water bodies as reported in the literature (Robards et al., 1994). With the exception of silicate whose molybdenum blue detection could be achieved at both ppb and ppm levels, the other species are detected only at the ppb level. From the scanned absorbance profiles, the extinction coefficient ratios and the concentration levels of the species in water bodies, the species which would seem to offer the greatest interferences are the arsenate, phosphate and silicate. In the case of polluted effluents, however, samples could contain increased levels of the vanadate and tungstate as well. 
TABLE 1

Concentration range (in ppb) as well as the major chemical form of occurrence of the species in natural fresh water and sea water.

\begin{tabular}{|l|cc|c|}
\hline \multirow{2}{*}{ Species } & \multicolumn{2}{|c|}{ Conc. range (ppb) } & \multirow{2}{*}{ Major species form(s) } \\
\cline { 2 - 3 } & Fresh water & Sea water & \\
\hline As & $0.2-230$ & $<0.5-3.7$ & $\mathrm{H}_{2} \mathrm{AsO}_{4}$; , organic \\
$\mathrm{P}$ & $5-1000$ & $<60-88$ & $\mathrm{H}_{2} \mathrm{PO}_{4}, \mathrm{HPO}_{4}{ }^{2-}, \mathrm{MgPO}_{4}{ }^{-}$ \\
$\mathrm{Si}$ & $500-60000$ & Trace-400 & $\mathrm{Si}(\mathrm{OH})_{4}$, colloid \\
V & $0.01-20$ & $<0.9-2.5$ & $\mathrm{H}_{2} \mathrm{VO}_{4}{ }^{-}, \mathrm{HVO}_{4}{ }^{2-}$ \\
W & $0.02-0.1$ & $0.001-0.7$ & $\mathrm{WO}_{4}{ }^{2-}$ \\
\hline
\end{tabular}

With the absorbance of a species being reasonably strong at the $\lambda_{\text {maxes }}$ of others (Figure 3 ), the $\lambda_{\text {maxes }}$ alone could not be used as the criterion for distinguishing between the species during the molybdenum blue detection. The surest way of removing any interference is by some sort of separation (isolation) prior to the molybdenum blue detection reaction. The initial unstable yellow-green baseline colour (reaction precursor) from the ascorbic acid-ammonium molybdate reagent mixture, formed prior to the formation of the molybdenum blue colour, would also affect the measurement process and therefore appropriate steps must be taken to address this.

\section{On-chip electrokinetic separation and detec- tion of the complex anions}

The on-chip electroosmotic flow (EOF) characteristics of solutions of the complex-anion water pollutants, namely orthophosphate, silicate, vanadate, tungstate and arsenate, and the optimum electric field strength for effective electrokinetic separation of these species have been evaluated on chip and reported (Doku et al., 2019), using the manifold given in Figure 2, which could be considered for future simultaneous on-chip micro capillary electrophoretic $(\mu \mathrm{CE})$ determination of the species. Using a positive Pt electrode dipping into the solution in a sample reservoir onchip and negative electrode in a borate-buffer waste collecting reservoir, and both electrodes connected by the buffer solution in a channel and an external power supply, the EOF characteristics, i.e., voltages applied across the electrodes, internal currents generated within the solutions and the flow of solution in the channels, at different pHs, were monitored. A conventional capillary electrophoresis, carried out in both positive and negative polarity and direct and indirect UV detection modes, using varied applied voltages (electric field strengths) was used to establish the optimum the electric field strength and retention times required for effective electrokinetic separation of the species. From the study it became clear that the species, tungstate, phosphate, arsenate, vanadate and silicate, were resolved under applied electric field, with the resolution between the tungstate and phosphate being the lowest. The elution time of a respective species was a compromise between the charge to size ratio of the species, the size/weight of the hydrated ion and the applied voltage. The best separation/resolution efficiencies were achieved with voltages between $5-10 \mathrm{kV}$ (field strengths between $77-154 \mathrm{~V} / \mathrm{cm}$ ), and the elution order of the species was found to be tungstate first, followed by phosphate, arsenate, orthovanadate and silicate in that order. At $5 \mathrm{kV}$, the retention times were found to be as follows: tungstate, 20.02; phosphate, 20.70; arsenate, 22.95; orthovanadate, 28.77; and silicate, 36.54 minutes. Thus, electrokinetic separation of the species has been confirmed possible, which could be carried out prior to detection or any analytical reaction and which will eliminate interferences between the species. The systematic procedure would offer a greater potential in terms of analyte detection in a chip-based device. 


\section{Conclusion}

The advancement so far achieved in phosphomolybdenum detection has been well reviewed. The review exposes the major challenges to be circumvented, the main one being interference from other molybdenum blue related species in water. The review will serve as a guide and reference point for future researchers in the area of advanced phosphate detection techniques yet to be designed. Lab-on-microchip based systems presents a promising area to explore in view of the numerous advantages over conventional systems.

\section{Recommendations}

(a) Carrying out the electrokinetic separation to complement the molybdenum blue detection in on-chip micro reactor systems would demand well designed procedures and appropriate manifolds. Two options are suggested here, as follows (Doku et al., 2016):

(i) Injecting the sample mixture into a stream of buffer in a longer injection/separation side-channel and applying the separation voltage to pre-separate the sample mixture into separate analyte bands in the side channel. This followed by normal FIA, involving sequential cross-mode injection of the already separated analyte bands into a continuous stream of the ascorbic acid-ammonium molybdate reagent mixture in the main reaction channel, with a short reagent run being carried out after each analyte band injection so as to segment the analyte bands in the reagent stream. This is followed, finally, by driving the background reagent stream pass the detection point. The appropriate on-chip manifold suggested for this procedure in any future work is given in Figure 4.

(ii) A reverse FIA method based on injecting the sample mixture into a stream of buffer in a longer main reaction channel and applying the electrical field to drive and separate the mixture into different analyte bands in the main reaction channel. This is carried out prior to a cross-mode injection of the ascorbic acid-ammonium molybdate reagent mixture just behind or in front of the separated analyte bands so that the injected mixture merges (interfaces) perfectly with the species band. A sequential drive of the separated analyte bands pass the reagent mixture injection point should alternate with timely injections of segments of the reagent mixture. The electric field polarity should be such that on resuming the main channel buffer flow towards the detector, after injections of the reagent mixture, the anionic species would migrate into and mix with the reagent mixture. The appropriate on-chip manifold suggested for this procedure is given in Figure 5. The former procedure (i) would, however, appear to be easier and is therefore more recommended.

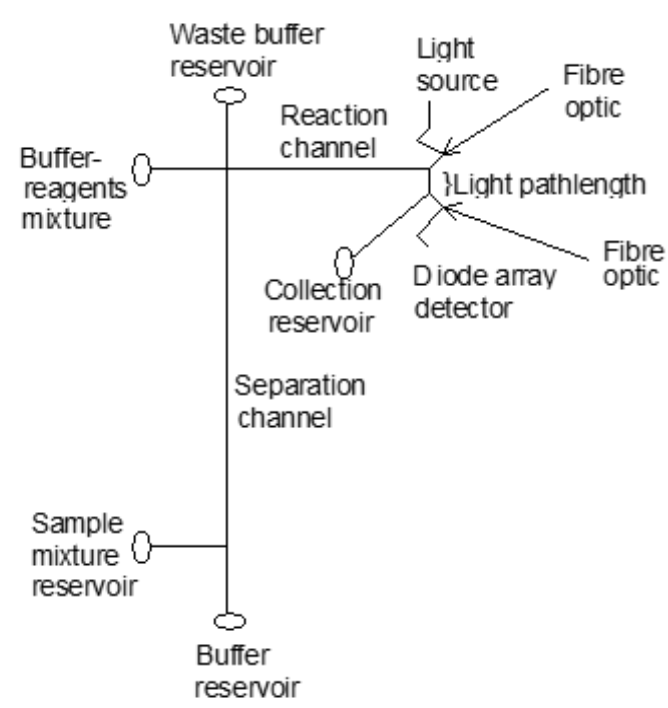

Fig. 4: On-chip manifold for procedure (i). 


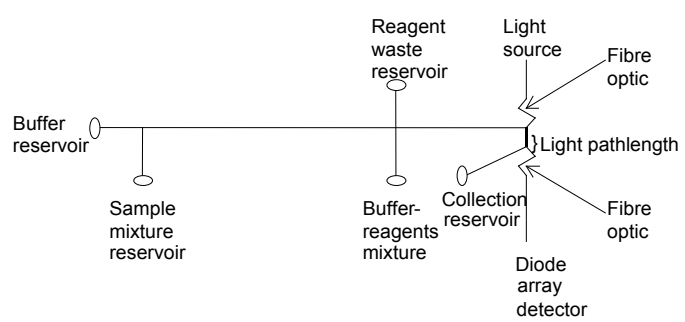

Fig. 5: On-chip manifold for procedure (ii). (b) Electrochemical detection, requiring no analytical reaction performance prior to detection, might be an easier way of detecting the species after separation, using micro electrodes integrated at the end of the separation channel (Figure 6) (Doku et al., 2019). The negative polarity mode is the more appropriate electric field operational mode to be used for the separation channel due to the shorter retention times it offers. This will however require the employment of a cationic surfactant in both the buffer and sample solutions to modify the capillary surface and reverse the electroosmotic flow from the cathode to the anode. The separation electric field strength to be employed should be within the range of 77 $-154 \mathrm{~V} / \mathrm{cm}$, with the applied voltage depending on the separation channel length.

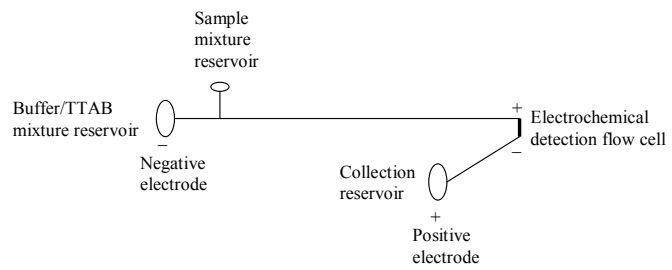

Fig. 6: On-chip CE manifold design for electrokinetic separation and detection of complex-anion water pollutants.

\section{References}

Antonisse, M. \& Reinhoudt, D. (1999) Potentiometric Anion Selective Sensors, Electroanalysis 11 (14), $1035-1048$.

Anzenbacher, P., Juriskova, K. \& Sessler, J. (2000) Second Generation Calixpyrrole Anion Sensors. J. Am. Chem. Soc. 122, 9350 - 9351.

Aoki, H., Hasegawa, K., Tohda, K. \& Umezawa, Y. (2003) Voltammetric detection of inorganic phosphate using ion channel sensing with self-assembled monolayers of a hydrogen bond forming receptor. Biosens. Bioelectron. 18, $261-267$.

Auroux, P. A., Lossifidis, D., Reyes, D. R. \& Manz, A. (2002) Micro total analysis systems. II. Analytical standard operations and applications, Anal. Chem. 74 (12), 2637.

Basset, J., Denney, R.c., Jeffery, G.H. \& Mendham, J. (1978) Vogel's Textbook of Quantitative Inorganic Analysis', 4th Ed., Longman, New York, p 390 and 731.

Beer, P. \& Cadman, J. (1999) Phosphate anion binding and luminescent sensing in aqueous solution by ruthenium (II) bipyridyl polyaza receptors. New J. Chem. 23, 347 - 349.

Beer, P., Cadman, J., Lloris, J., Martínez-Máñez, R., Padilla, M., Pardo, T., Smith, D \& Soto, J. (1999) Selective electrochemical recognition of sulfate over phosphate and phosphate over sulfate using polyaza ferrocene macrocyclic receptors in aqueous solution. J. Chem. Soc., Dalton Trans. 127 - 133.

Beer, P., Chen, Z., Drew, M., Johnson, A., Smith, D. \& SPencer, P. (1996) Transition metal cation and phosphate anion electrochemical recognition in water by new polyaza ferrocene macrocyclic ligands. Inorg. Chim. Acta 246, 143 -150 .

Beer, P., Drew, M. G. \& Smith, D. (1997) Selective electrochemical recognition of bidentate anionic guests in competitive solvents using novel ferrocenyl thiourea and guanidinium receptors. J. Organomet. Chem. 543, 259 - 261. 
Beer, P., Drew, M. G., Smith, D. \& Stokes, S. (1995) Quantitative and Structural Investigations of Hydrogen Bonding Interactions in Anion Binding of Mono- and 1,1'Bis-substituted Aryl Cobaltocenium Receptors. J. Chem. Soc., Dalton Trans. $403-408$.

Bello, M. A. \& Gonzalez, A. G. (1996) Determination of Phosphate in Cola Beverages Using Nonsuppressed Ion Chromatography: An Experiment Introducing Ion Chromatography for Quantitative Analysis, J. Chemical Education 73 (12), 1174.

Berchmans, S., Karthikeyan, R., Gupta, S., Poinern, G., Issa, T. \& Singh, P. (2011) Glassy carbon electrode modified with hybrid films containing inorganic molybdate anions trapped in organic matrices of chitosan and ionic liquid for the amperometric sensing of phosphate at neutral pH. Sens. Actuators, B 160, $1224-1231$.

Carey, C. M. \& Riggan, W. B. (1994) Cyclic polyamine ionophore for use in a dibasic phosphate selective electrode. Anal. Chem. 66, $3587-$ 3591 .

Cheng, W.-L., Sue, J.-W., Chen, W.-C., Chang, J.-L. \& ZeN, J.-M. (2010) Activated Nickel Platform for Electrochemical Sensing of Phosphate. Anal. Chem. 82 (3), $1157-1161$.

Clark, J., Simpson, H., Bopp, R. \& Deck, B. (1992) Geochemistry and loading history of phosphate and silicate in the Hudson estuary, Estuarine, Coastal and Shelf Sci. 34, 213.

Clinton-Bailey, G. S., Grand, M. M., Beaton, A. D., Nightingle, A. M., Owsianka, D. R., Slavik, G. J., Connelly D. P., Cardwell C. L. \& Mowlem, M. C. (2017) A lab-on-chip analyzer for in situ measurement of soluble reactive phosphate: improved phosphate blue assay and application to fluvial monitoring. Environmental science \& technology 51(17), 9989 $-9995$.

Costantini, F., Benetti, E. M., Reinhoudt, D. N., Huskens, J., Vancso, G. J. \& Verboom, W. (2010) Enzyme-functionalized polymer brush films on the inner wall of silicon-glass micro- reactors with tunable biocatalytic activity, $L a b$ Chip 10, 3407.

Costantini, F., Benetti, E. M., Tiggelaar, R. M., Gardeniers, H. J. G. E., Reinhoudt, D. N., Huskens, J., Vancso, G. J. \& Verboom, W. (2010) Gallium-containing polymer brush film as efficient supported Lewis acid catalyst in a glass microreactor, Chem. Eur. J. 16, 12406.

Daykin, R. N. C. \& Haswell, S. J. (1995) Development of a micro flow injection manifold for the determination of orthophosphate, Anal. Chim. Acta. 313, 155.

Demedina, H. L., Gutierrez, E., Devargas, M. C., Gonzalez, G., Marin, J. \& Andueza, E. (1996) Determination of phosphate and sulphite in natural waters in the presence of high sulphate concentrations by ion chromatography under isocratic conditions, J. Chromatogr. A 739, $1-2,207$.

Deetz, M. \& Smith, B. (1998) Heteroditopic Ruthenium (II) Bipyridyl Receptor with Adjacent Saccharide and Phosphate Binding Sites. Tetrahedron Lett. 39, 6841 - 6844.

Doku, G. N. \& Haswell, S. J. (1999) Further studies into the development of a micro-FIA $(\mu$ FIA $)$ system based on electroosmotic flow for the determination of phosphate as orthophosphate, Anal. Chim. Acta. 382, 1.

Doku G. N., Haswell S. J. \& Mccreedy T. (2016) Preliminary Studies into the Degree of Molybdenum Blue Interference among Complex-Anion Water Pollutants, Towards On-Chip Micro-FIA Simultaneous Spectrophotometric Determination', Central Inquiry, 2(1), 120.

Doku G. N., Haswell S. J. \& Mccreedy T. (2019) OnChip Electroosmotic Flow Characteristics and Electric Field strength for the Electrokinetic Separation of Complex-Anion Water Pollutants, Towards Possible On-Chip Simultaneous Determination, Journal of The Ghana Science Association 18 (1), 7 - 13.

Du, J., Wang, X., Jia, M., Li, T., Mao, J. \& Guo, Z. (2008) Recognition of Phosphate Anions in 
Aqueous Solution by a Dinuclear Zinc (II) Complex of a Cyclen Tethered Terpyridine Ligand. Inorg. Chem. Commun. 11, $999-1002$.

Ejhieha, A. \& Masoudipoura, N. (2010) Application of a new potentiometric method for determination of phosphate based on a surfactant-modified zeolite carbon-paste electrode (SMZCPE). Anal. Chim. Acta 658, 68 - 74.

Engblom, S. (1998) The phosphate sensor. Biosens. Bioelectron. 13, 981 - 994.

Fabbrizzi, L., Marcotte, N., Stomeo, F. \& Taglietti, A. (2002) Pyrophosphate Detection in Water by Fluorescence Competition Assays: Inducing Selectivity through the Choice of the Indicator. Angew. Chem., Int. Ed. 41 (20), 3811 -3814 .

Fogg, A. \& Bsebsu, N. (1981). Differential-pulse Voltammetric Determination of Phosphate as Molybdovanadophosphate at a Glassy Carbon Electrode and Assessment of Eluents for the Flow Injection Voltammetric Determination of Phosphate, Silicate, Arsenate and Germanate. Analyst 106, 1288 - 1295.

Frost, C. G. \& Mutton, L. (2010) Heterogeneous catalytic synthesis using microreactor technology, Green Chem. 12, 1687 - 1703.

Galceran, M. T. \& Diez, M. (1994) Column-switching techniques in the analysis of phosphate by ion chromatography, J. Chromatogr. A 675 (1 $-2), 141$.

Ganjali, M., Hosseini, M., Memari, Z., Faridbod, F., Norouzi, P., Goldooz, H. \& BadieI, A. (2011) Selective recognition of monohydrogen phosphate by fluorescence enhancement of a new cerium complex. Anal. Chim. Acta 708, 107 -110 .

Ganjali, M., Norouzi, P., Ghomi, M. \& SalavaTI-Niasari, M. (2006) Highly selective and sensitive monohydrogen phosphate membrane sensor based on molybdenum acetylacetonate. Anal. Chim. Acta 567, 196 - 201.
Gáspár, A., Bácsi, I., Garcia, E. F., Braun, M., \& GoMEZ, F. A. (2009) Application of external micro-spectrophotometric detection to improve sensitivity on microchips. Analytical and bioanalytical chemistry 395 (2), 473 - 478.

Glazier, S. \& ARnold, M. (1988) Phosphate selective polymer membrane electrode. Anal. Chem. 60, $2540-2542$.

Greenway, G. M., Haswell, S. J. \& Petsul, P. H. (1999) On-chip spectrophotometric determination of nitrite, Anal. Chim. Acta 387, 1.

HAMACHI, I. (2009) Patent No. 7,521,250 B2. USA.

Han, M. \& Kim, D. (2002) Naked-Eye Detection of Phosphate Ions in Water at Physiological $\mathrm{pH}$ : A Remarkably Selective and Easy-To-Assemble Colorimetric Phosphate-Sensing Probe. Angew. Chem., Int. Ed. 41 (20), 3809 - 3811.

Haswell, S. J. (1997) Development and Operating Characteristics of Micro Flow Injection Analysis Systems Based on Electroosmotic Flow, Analyst 122, 1R.

Irfan, M., Glasnov, T. N. \& Kappe, C. O. (2011) Heterogeneous Catalytic Hydrogenation Reactions in Continuous-Flow Reactors, ChemSusChem 4, $300-316$.

Jonca, J., Fernandez, V., Thouron, D., Paulmier, A., Graco, M. \& Garcon, V. (2011). Phosphate determination in seawater: Toward an autonomous electrochemical method. Talanta 87, $161-167$.

Khaled, E., Hassan, H., Girgis, A. \& Metelka, R. (2008) Construction of novel simple phosphate screen-printed and carbon paste ion selective electrodes. Talanta 77, 737 - 743.

Kugimiya, A. \& Kohara, K. (2009) Biomimetic sensor for cAMP using an ion-sensitive field-effect transistor. Mater. Sci. Eng., C 29, 959 - 962.

Kumar, A., Mehtab, S., Singh, U., Aggarwal, V. \& Singh, J. (2008) Tripodal Cadmium Complex and Macrocyclic Ligand Based Sensors for 
Phosphate Ion Determination in Environmental Samples. Electroanalysis 20, 1186 - 1193.

Kumar, P., Kim, D.-M., Hyun, M.-H. \& Shim, Y.-B. (2010). An all-solid-state monohydrogen phosphate sensor based on a macrocyclic ionophore. Talanta 82, 1107 - 1112 .

Lee, J.-H., Lee, W., Bishop, P. \& Papautsky, I. (2009) A cobalt-coated needle type microelectrode array sensor for in situ monitoring of phosphate. J. Micromech. Microeng. 19 (Feb), 1 -6.

Le Goff, T., Braven, J., Ebdona, L. \& Scholefield, D. (2004) Phosphate-selective electrodes containing immobilised ionophores. Anal. Chim. Acta 510, 175 - 182.

Lyddy-Meaney A. J, Ellis P. S., Worsfold P. J., ButLer E. C. \& Mckelvie I. D (2002) A compact flow injection analysis system for surface mapping of phosphate in marine waters. $\mathrm{Ta}$ lanta 58 (6), 104 - 353.

Liu, D., Chen, W.-C., Yang, R.-H., Shen, G.-L. \& Yu, R.-Q. (1997) Polymeric membrane phosphate sensitive electrode based on binuclear organotin compound. Anal. Chim. Acta 338, $209-$ 214.

Liu, G. \& Lin, Y. (2005) Electrochemical sensor for organophosphate pesticides and nerve agents using zirconia nanoparticles as selective sorbents. Anal. Chem. 77, $5894-5901$.

Lin, X., Wu, X., Zenghong, X. \& Wong, K.-Y. (2006) PVC matrix membrane sensor for fluorescent determination of phosphate. Talanta 70, 32 36.

Liu, W., Li, X., Song, M. \& Wu, Y. (2007) A novel dibasic phosphate-selective electrode based on Ferrocene-bearing macrocyclic amide compound. Sens. Actuators, B 126, $609-615$.

Ma, L., Tsuboi, T., Hattori, T., Oshima, M., TAKayanagi, T. \& Motomizu, S. (1999) On-site chemical analysis for nitrogen, phosphorus and sulfur compounds in the environment: sensitive determination by microflow injection method. J Flow Inject Anal 16, 79 - 88.
Mattusch, J. \& WennRich, R. (1996) Elimination of sulfate interferences in the Chromatographic determination of o-phosphate using liquid-liquid extraction, J. Analytical Chemistry 356 (5), 335 .

Meruva, R. \& Meyerhoff, M. (1996) Mixed Potential Response Mechanism of Cobalt Electrodes toward Inorganic Phosphate. Anal. Chem. 68 (13), $2022-2026$.

Modi, N., Patel, B., Patel, M. \& Menon, S. (2011) Novel monohydrogenphosphate ion-selective polymeric membrane sensor based on phenyl urea substituted calix [4] arene. Talanta 86, $121-127$.

Midgley, D. (1986) Sulphate and phosphate ion-selective electrodes. Ion-Sel. Electrode Rev. 8, $3-54$.

Motomizu, S., Susanto, J. P., Oshima, M., Mikasa, H. \& HoRI, Y. (1995) Ultra-trace determination of phosphate ion based on filtration-dissolution and flow-through spectrophotometric measurement, Analytical Sciences 11 (1), 155.

Murphy, J. \& Riley, J. (1962) A modified single solution method for the determination of phosphate in natural waters, Anal. Chim. Acta $31-36$.

Natalie, G. W. \& Mccreedy, T (2000) On-chip catalysis using a lithographically fabricated glass microreactor - the dehydration of alcohols using sulfated zirconia, Chem. Commun., 733 -734 .

Nishizawa, S., Yokobori, T., Kato, R., Yoshimoto, K., Kamaishi, T. \& Teramae, N. (2003) Hydrogen-bond forming ionophore for highly efficient transport of phosphate anions across the nitrobenzene-water interface. Analyst 128, $663-669$.

Ojida, A., Mito-Oka, Y., Inoue, M. \& Hamachi, I. (2002) First Artifical receptors and Chemosensors toward Phosphorylated Peptide in Aqueous Solution. J. Am. Chem. Soc. 124, $6256-6258$. 
Oлida, A., Мito-OKa, Y., Sada, K. \& Hamachi, I. (2004) Molecular Recognition and Fluorescence Sensing of Monophosphorylated Peptides in Aqueous Solution by Bis(zinc(II) dipicolylamine)-Based Artificial Receptors. J. Am. Chem. Soc. 126, $2454-2463$.

Quintana, J. C., Idrissi, L., Palleschi, G., Albertano, P., Aminec, A., El Rhazi, M. \& Mosconea, D. (2004) Investigation of amperometric detection of phosphate, application in seawater and cyanobacterial biofilm samples. Talanta 63, $567-574$.

Rahman, M. A., Park, D.-S., Chang, S.-C., Mcneil, C. J. \& SHim, Y.-B. (2006). The biosensor based on the pyruvate oxidase modified conducting polymer for phosphate ions determinations. Biosens. Bioelectron. 21, $1116-1124$.

Reyes, D. R., Lossifidis, D., Auroux, P. A. \& Manz, A. (2002) Micro Total Analysis Systems. I. Introduction, Theory, and Technology, Anal. Chem. 74 (12), 2623.

Robards, K., Mckelvie, I. D., Benson, R. L., WorsFOLD, P.J., Blundell, N.J. \& CASEy, H. (1994) Determination of carbon, phosphorus, nitrogen and silicon species in waters, Analytica Chimica Acta, 287, 147.

Ruzicka J. \& Hanson E. H. (1988) Flow Injection Analysis, 2nd. Ed., Willey, p1.

Sessler, J., Davis, J., Kral, V., Kimbrough, T. \& Lynch, V. (2003) Water Soluble Sapphyrins: Potential Fluorescent Phosphate Anion sensors. Org. Biomol. Chem. 1, $4113-4123$.

Sun, H., Scharff-Poulsen A. M., Gu, H., Jakobsen, I., Kossmann J. M., Frommer W. B. \& Almdal, K. (2008) Phosphate Sensing by Fluorescent Reporter Proteins Embedded in Polycrylamide Nanoparticles. ACS Nano 2 (1), $19-24$.

TAfesse, F. \& Enemchukwu, M. (2011) Fabrication of a New Solid State Phosphate Selective Electrodes for Environmental Monitoring. Talanta 83, $1491-1495$.
Tsagkatakis, I., Chaniotakis, N., Altmann, R., Jurkschat, K., Willem, R., Martins, J., Qin, Y. \& BAKKeR, E. (2001) Phosphate-binding characteristics and selectivity studies of bifunctional organotin carriers. Helv. Chim. Acta 84, 1952 -1961 .

Tobey, S. \& Anslyn, E. (2003) Determination of Inorganic Phosphate in Serum and Saliva Using a Synthetic Receptor. Org. Lett. 5 (12), 2029 $-2031$.

Udnan, Y., Mckelvie, I., Grace, M., Jakmunee, J. \& GRUDPAN. (2005) Evaluation of on line preconcentration and inflow-injection amperometery for phosphate determination in fresh and marine waters. Talanta 66, $461-466$.

Verpoorte, E. M. J., Van Der Schoot, B. H., Jeanneret, S., Manz, A. \& De Rooij, N. F. (1994) Silicon based chemical microsensors and micro-systems, In interfacial design and chemical sensing, Amer. Chem. Soc. symposium series, Ch21, p244.

Villalba, M., Mckeegan, K., Vaughan, D., Cardosi, D. \& DAvis, J. (2009) Bioelectroanalytical determination of phosphate - A review. J. Mol. Catal. B: Enzym. 59, $1-8$.

Warwick, C., Guerreiro, A. \& Soares, A. (2013) Sensing and analysis of soluble phosphates in environmental samples: A review. Biosensors and Bioelectronics 41, 1-11.

Wegner, J., Ceylan, S. \& Kirschning, A. (2011) Ten key issues in modern flow chemistry, Chem. Commun. 47, 4583 - 4592.

Wiles, C. \& Watts, P. (2012) Continuous flow reactors: a perspective, Green Chem. 14, 38 - 54 .

Xiao, D., Yuan, H.-Y., Li, J. \& Yu, R.-Q. (1995) Surface-Modified Cobalt-Based Sensor as a Phosphate Sensitive Electrode. Anal. Chem. 67, $288-291$.

Yaqoob, M., Nabi, A. \& Worsfold, P. J. (2004) Determination of nanomolar concentrations of phosphate in freshwaters using flow injection 
with luminol chemiluminescence detection. Anal. Chim. Acta 510, $213-218$.

Yeh, P., Yeh, N., Lee, C.H., \& Ding, T. J. (2017) Applicationsof LEDs in optical sensors and chemical sensing device for detection of biochemicals, heavy metals, and environmental nutrients. Renewable and Sustainable Energy Reviews 75, 461 - 468.

Zhang, Z., Jaffrezic-Renault, N., Bessueille, F., Leonard, D., Xia, S., Wang, X., Chen, L. \&
ZhaO, J. (2008) Development of a conductometric phosphate biosensor based on tri-layer maltose phosphorylase composite films. Anal Chim Acta. 615, 73 - 79.

Zou, Z., HAN, J., JANG, A., BishoP, P. \& AHN, C. (2007). A disposable on-chip phosphate sensor with planar cobalt microelectrodes on polymer substrate. Biosens. Bioelectron. 22, 1902 - 1907.

Received 03 Jun 19; revised 26 Jun 20. 